\title{
Philippe ARTIÈRES, La vie écrite. Thérèse de Lisieux,
} biographie

Éditions Les Belles Lettres, 2011, coll. « L'histoire de profil », 241 pages.

\section{Cécile Dauphin}

\section{CpenEdition}

Journals

Édition électronique

URL : https://journals.openedition.org/clio/10432

DOI : 10.4000/clio.10432

ISSN : 1777-5299

Éditeur

Belin

Édition imprimée

Date de publication : 31 décembre 2011

ISBN : 978-2-8107-0170-4

ISSN : $1252-7017$

\section{Référence électronique}

Cécile Dauphin, «Philippe artières, La vie écrite. Thérèse de Lisieux, biographie », Clio. Femmes, Genre, Histoire [En ligne], 34 | 2011, mis en ligne le 15 décembre 2011, consulté le 23 avril 2022. URL : http:// journals.openedition.org/clio/10432; DOI : https://doi.org/10.4000/clio.10432

Ce document a été généré automatiquement le 23 avril 2022.

Tous droits réservés 


\section{Philippe ARTIÈrES, La vie écrite. Thérèse de Lisieux, biographie}

Éditions Les Belles Lettres, 2011, coll. «L'histoire de profil », 241 pages.

\section{Cécile Dauphin}

\section{RÉFÉRENCE}

Philippe ARTIÈRES, La vie écrite. Thérèse de Lisieux, biographie, Éditions Les Belles Lettres, 2011, coll. « L'histoire de profil », 241 pages.

1 En proposant une biographie de Thérèse de Lisieux, Philippe Artières entend apporter une contribution à l'histoire sociale de l'écriture, en particulier à la manière dont l'écriture est partagée entre différents milieux. Son enquête couvre ainsi un large spectre culturel qui va des pratiques de routine ou des actes exceptionnels jusqu'à la façon dont ces écrits sont regardés et commentés par les experts (policiers, médecins ou scientifiques). Clairement inscrite dans le cadre d'une anthropologie historique de l'écriture, la biographie de la petite sainte (née en 1873 et décédée en 1897) devient une sorte de fil d'Ariane pour cheminer à travers la société à la fin du XIX siècle et la multiplicité des formes graphiques qui se diffusent alors.

L'organisation même de l'ouvrage participe du déplacement revendiqué par l'historien dans le genre biographique. Originalité annoncée dès la couverture. Empruntant à la sainte la posture et les attributs (roses et crucifix), au mysticisme les couleurs de feu, à l'enracinement local la graphie d'un LISIEUX sur-imprimé, l'icône de la carmélite apparaît voilée dans un brouillard de pixels, tel un zoom fractionnant l'image. Au-delà du style Pop Art de cette présentation, l'agencement des chapitres vient conforter cette idée d'expérimentation dans le va-et-vient entre les archives et dans l'effet puzzle de la juxtaposition des documents. Ainsi sont placés en «ouverture " un mémoire autobiographique - d'une violence inouïe - et une prière solennelle, rédigés par un jeune homme sanguinaire (né en 1888). Ce détour invite à penser ensemble la sainte et le criminel, ou plutôt l'étrangeté de l'écrit mystique, qu'il soit saint ou criminel. Vient 
ensuite une «liste des protagonistes par ordre d'apparition». À n'en pas douter, l'historien joue sur la théâtralisation des textes produits par et autour de Thérèse. Cette biographie qui se veut "à contre emploi » se trouve ainsi installée à un carrefour improbable où le personnage principal est à la fois auteur et objet d'un assemblage discursif, où la parenté des formes graphiques place la carmélite à l'ombre de l'infâme, comme exact envers du criminel, ordinaire et inconnue jusqu'à sa mort. Le feuilletage des chapitres fait naviguer le lecteur entre les couches de l'énorme corpus thérésien et divers îlots d'écritures contemporaines.

3 Tous ces indices offerts au premier regard soulignent l'attention portée à la matérialité des textes et des objets, la façon particulière de capturer le sujet, de l'inscrire dans une matrice de relations scripturaires, de donner sens à une mosaïque d'écrits. Du genre biographique, l'historien respecte les principes: la focalisation sur un personnage et sur ses points d'ancrage dans le temps et l'espace social, le décryptage des traces, la disposition chronologique des chapitres permettant de repasser le film déjà connu de la vie proprement dite de Thérèse. L'originalité de la démarche tient dans l'exercice de contextualisation et la quête de résonances qui cherchent à saisir chacun des gestes dans un réseau d'objets et de pratiques pouvant se faire écho ou s'entrechoquer. Ainsi, les " petites écritures » de Thérèse tout au long de son enfance et de sa vie religieuse, qui accumulent copies, dessins, listes, découpages, calligraphies, etc., apparaissent conformes à l'art d'agencer images et textes alors inculqué dans les familles, les écoles et les couvents. Mais ces prises d'écriture plus ou moins contraintes à différents moments de sa courte vie sont autant d'événements qui modifient radicalement son existence. Qu'il s'agisse de la copie du Credo avec son propre sang ou de la rédaction des récits autobiographiques, tout en répondant à l'attente de son entourage Thérèse trouve par là moyen de se mettre en scène et de construire sa "cause " en quelque sorte. Toutes ces pratiques sont rapprochées de celles rencontrées chez certains criminels comme Joseph Vacher, dit le tueur de bergers, également graphomane, conscient du pouvoir de l'écrit dans l'enquête menée sur son crime. Il se mure littéralement derrière une citadelle d'écritures, tapisse les murs de la prison de sentences pareilles à celles du carmel, développe surtout une intense activité épistolaire, cherchant à coller au plus près des savoirs médicaux. La connaissance précoce que Thérèse acquiert des vies de saints lui donne la possibilité de se conformer aux normes de la sainteté. Par ses écrits et la manière d'en user, elle se fabrique comme sainte. Spécialiste des écritures criminelles, l'A. souligne que, dans les deux cas, les dispositifs incitatifs sont similaires et répondent d'une façon ou d'une autre à des jeux de pouvoir qui inscrivent le récit de soi comme une étape obligée pour accéder à l'expérience mystique ou à la demande de justice.

4 Au lendemain de la mort de la jeune carmélite, la prophétie de la pluie de roses énoncée de son vivant se réalise avec la publication de ses textes. Minutieusement orchestrée par le couvent, l'opération connaît un énorme succès et suscite à son tour un véritable phénomène de concentration d'écrits: durant des années, des milliers de lettres convergent vers la petite cité normande et alimentent un périodique (Annales de sainte Thérèse de Lisieux) qui devient une sorte de grand manifeste collectif relatant des conversions, des effets de lecture, le pouvoir de protection de ces écrits psychologique, morale et physique -, en particulier chez les poilus qui les portent sur le cœur tel un bouclier. Le biographe de Thérèse ne manque pas de rapprocher aussi cette 
pluie d'écrits des lettres anonymes suscitées par la vague d'attentats anarchistes qui sèment la panique en France au début des années 1890.

Suivant la même démarche, la question des expertises en écriture dans l'affaire Dreyfus est évoquée pour interroger la matérialité du corpus thérésien, à savoir les opérations de qualification, de classement, de transcription et d'édition qui ont constitué les écrits de Thérèse en « archives de la sainte ». Dans cette histoire centenaire (1898-1998), l'A. distingue trois épisodes: d'abord la publication de l'Histoire d'une âme, occasion de retravailler le récit autobiographique en partie composé pour préparer la rédaction de la notice nécrologique envoyée traditionnellement à tous les carmels ; puis le procès en béatification, procédure lancée en 1909, qui entraîne une série de transcriptions des cahiers et une entreprise de captation des paroles de la sainte; enfin l'établissement des Oeuvres complètes qui mobilise plusieurs maisons d'édition et instaure un véritable débat scientifique sur les questions d'exhaustivité et d'exactitude. La laborieuse fabrique du corpus participe de l'avènement des archives comme composante de l'œuvre d'un auteur, comprenant jusqu'au moindre billet ou brouillon.

6 Au royaume de l'hagiographie, Thérèse de Lisieux occupe une place incontestée : elle est considérée comme fille spirituelle de Jean de la Croix et de Thérèse d'Avila, précocement canonisée, puis élevée au titre de 33 docteur de l'Église (en 1997 par le pape Jean-Paul II), vénérée partout dans le monde bien au-delà de sa Normandie natale. Le pas de côté auquel nous invite Ph. Artières débouche finalement sur un autre projet que la biographie, aussi stimulant qu'audacieux. Familier de la clinique des écritures, des vies coupables, des formes multiples de l'épistolaire, lettres, billets anonymes ou confessions, l'historien excelle à tirer de ce registre polymorphe de nouvelles combinaisons pour questionner l'histoire de la culture écrite, pointant précisément le grain de sable qui résiste et enraie la logique d'un système. Dans le grand panoptique graphique mis en place au XIX ${ }^{\mathrm{e}}$ siècle, notamment autour des criminels et des saints, le cas de Thérèse de Lisieux devient paradigme pour éprouver les notions de l'ordinaire, d'événement, de matérialité, d'environnement graphique. Autrement dit, la biographie consiste ici à «désingulariser» l'extraordinaire destin de la petite carmélite en montrant, textes à l'appui, son caractère profondément ordinaire.

\section{AUTEURS}

\section{CÉCILE DAUPHIN}

Cécile Dauphin est ingénieure de recherche au CNRS (Centre de recherches historiques - École des hautes études en sciences sociales). Elle a développé des recherches dans deux champs principaux : l'histoire socio-culturelle des pratiques épistolaires et l'histoire des femmes (femmes seules, violence, séduction). Elle travaille avec Danièle Poublan à la mise en ligne d'une correspondance familiale (http://correspondancefamiliale.ehess.fr/). dauphin@ehess.fr 